\title{
A SCHROEDER-BERNSTEIN THEOREM FOR PROJECTIONS ${ }^{1}$
}

\section{ARNOLD LEBOW}

The purpose of this note is to give the algebraic part of a proof for a result from the theory of von Neumann algebras.

Consider a ring with involution $\left(^{*}\right)$ satisfying $(a+b)^{*}=a^{*}+b^{*}$ and $(a b)^{*}=b^{*} a^{*}$. An idempotent $e$ is called a projection if it is selfadjoint $\left(e=e^{*}\right)$. The set $P$ of all projections is partially ordered by the relation $\leqq$, defined by setting $e \leqq f$ whenever $e f=e$. A pair of projections, $e$ and $f$, are said to be equivalent $(e \sim f)$ if there is an element $v$ such that $v v^{*}=e$ and $v^{*} v=f$. A new relation $\prec$ is defined by $e \prec f$ if $e \sim g \leqq f$.

THEOREM. If the partially ordered set $(P, \leqq)$ is a complete lattice, then $e \prec f$ and $f \prec e$ imply $e \sim f$.

In order to make this paper self contained the following well-known results are included.

Lemma 1. If $e_{i} \sim f_{i}(i=1,2), e_{1} e_{2}=0$, and $f_{1} f_{2}=0$ then $e_{1}+e_{2} \sim f_{1}+f_{2}$.

Proof. By assumption there exist $v_{i}$ such that $v_{i}^{*} v_{i}=f_{i}$ and $v_{i} v_{i}^{*}=e_{i}$. Let $w=v_{1} v_{1}^{*} v_{1}+v_{2} v_{2}^{*} v_{2}$, then $w w^{*}=e_{1}+e_{2}$ and $w^{*} w=f_{1}+f_{2}$.

Lemma 2. An order preserving map on a complete lattice has a fixed point.

Proof. Let $\phi$ be the map and $p=\sup E$ where

$$
E=\{g \mid g \leqq \phi(g)\} .
$$

For $g$ in $E, g \leqq p$ so that $g \leqq \phi(g) \leqq \phi(p)$, therefore $p \leqq \phi(p)$. It follows that $\phi(p) \leqq \phi(\phi(p))$, implying $\phi(p)$ is in $E$, hence $\phi(p) \leqq p$.

Proof of THE TheOREM. By assumption there are elements $v$ and $w$ such that $w^{*} w \leqq f, w w^{*}=e, v^{*} v \leqq e$ and $v v^{*}=f$. For $g \leqq f$ define

$$
\phi(g)=f-w^{*}\left(e-v^{*} g v\right) w .
$$

Direct computation shows that $h \leqq g \leqq f$ implies $v^{*} h v \leqq v^{*} g v \leqq e$ and $f-g \leqq f-h$. Now it can be seen that $\phi$ is the composition of four maps of which two are order preserving and two are order reversing. Thus it follows that $\phi$ is order preserving on the complete lattice $[0, f]$, so that by Lemma 2 there is a projection $p \leqq f$ such that

Received by the editors November 18, 1966.

1 Supported in part by NSF Grant No. GP-5436. 


$$
p=f-w^{*}\left(e-v^{*} p v\right) w .
$$

The elements $p v$ and $\left(e-v^{*} p v\right) w$ implement the equivalences

$$
p \sim v^{*} p v \text { and } f-p \sim e-v^{*} p v .
$$

Now it follows from Lemma 1 that $f \sim e$.

Von Neumann algebras, $W^{*}$-algebras, $A W^{*}$-algebras and Baer rings are examples of rings with a complete lattice of projections.

The author wishes to express his appreciation to Bernie Russo whose seminar inspired this paper.

University of California, Irvine

\section{ON THE FOURIER INVERSION THEOREM FOR $R^{1}$}

\section{IAN RICHARDS ${ }^{1}$}

The following is an elementary "noncomputational" proof of the Fourier inversion theorem for tempered distributions on $R^{1}$. The proof does not generalize so easily to $R^{n}$, but the inversion theorem for $R^{n}$ can be deduced from that for $R^{1}$.

To get the inversion theorem for tempered distributions it is suffcient, by duality, to have a proof for the space $D$ of test functions (i.e. functions $\phi \in C^{\infty}$ such that $\phi^{(m)}(x)=O\left(|x|^{-N}\right)$ for all $m, N \geqq 0$ as $x \rightarrow \pm \infty)$. It is also sufficient to consider only the point $x=0$.

THEOREM. There exists a universal constant $K$ such that

(1) $\int_{-\infty}^{\infty} \hat{\phi}(t) d t=K \phi(0)$ for all $\phi \in D$.

The value of $K(K=2 \pi)$ must be determined, as usual, by substituting some particular function $\phi$. By linearity, (1) is equivalent to the following:

(2) $\phi(0)=0$ implies $\int_{-\infty}^{\infty} \hat{\phi}(t) d t=0$.

Proof of (2). Since $\phi(0)=0, \psi(x) \equiv(\phi(x) / x) \in C^{\infty}$. By direct computation, since $\phi(x)=x \psi(x), \hat{\phi}(t)=i(d / d t) \hat{\psi}(t)$. Then, since $\hat{\psi}$ is also a test function (direct verification), $\int_{-\infty}^{\infty} \hat{\phi}(t) d t=i[\hat{\psi}(\infty)-\hat{\psi}(-\infty)]$ $=0$. Q.E.D.

UNIVERSity OF MinNESOTA

Received by the editors October 27, 1966.

1 This work was partially supported by NSF Grant GP 4033. 